\title{
Ictiofauna do Ribeirão Limoeiro, Bacia do Rio Cachoeira, BA
}

\author{
Márcia Emília de Jesus Trindade ${ }^{1,4}$, Mauricio Cetra ${ }^{1,2,4}$ \& Ricardo Jucá-Chagas ${ }^{1,3}$ \\ ${ }^{1}$ Programa de Pós-graduação em Sistemas Aquáticos Tropicais, Departamento de Ciências Biológicas, \\ Universidade Estadual de Santa Cruz - UESC, Rod. Ilhéus-Itabuna, Km 16, \\ CEP 45662-000, Ilhéus, BA, Brasil \\ ${ }^{2}$ Curso de Ciências Biológicas, Universidade Federal de São Carlos - UFSCar, \\ Rod. João Leme dos Santos, Km 110, SP 264, CEP 18052-780, Sorocaba, SP, Brasil \\ ${ }^{3}$ Universidade Estadual do Sudoeste da Bahia - UESB, Rua José Moreira Sobrinho, s/n, \\ Jequiezinho, CEP 45200-000, Jequié, BA, Brasil \\ ${ }^{4}$ Autores para correspondência: Mauricio Cetra,e-mail: mcetra@ufscar.br \\ Márcia Trindade, e-mail: mejtrindade@hotmail.com
}

TRINDADE, M.E.J., CETRA, M. \& JUCÁ-CHAGAS, R. Ichthyofauna of the Ribeirão Limoeiro, Cachoeira River Basin, BA. Biota Neotrop. 10(4): http://www.biotaneotropica.org.br/v10n4/en/abstract?article+bn02010042010.

\begin{abstract}
The streams of the Eastern Basin are characterized by being small, somewhat complex and endemic fish fauna due to geographical isolation. In this context, a study of species distribution and community structure of stream fish Limoeiro, BA along a spatial gradient in one year, using electrofishing as the collection method. Twenty one fish species were collected totalizing 2264 specimens in four points: two points near the head (P1A and $\mathrm{P} 1 \mathrm{M})$, one in the middle course (P2) and another near the mouth (P3). Characiformes and Siluriformes were the orders more important among the four registered. Some species occurred exclusively at the head (Astyanax sp. and Trichomycterus cf. bahianus) and other near the mouth (Leporinus sp. and Callichthys callichthys). On the other hand, Characidium zebra, Parotocinclus cf. cristatus, Poecilia vivipara, Astyanax sp.1, Rhamdia sp. and Poecilia reticulata occurred all sampling points. Was collected one specie the "status" of vulnerable (Nematocharax venustus) and two exotics (Tilapia rendalli and Poecilia reticulata). About the community structure, the values of the index of richness of "Margalef" and of the index of diversity of "Shannon" were greater in this point P3, then this point were significantly lower value of "Berger-Parker" dominance index. The point with riparian vegetation preserved $(\mathrm{P} 1 \mathrm{M})$ showed a lower average water temperature and the points near the head (P1A and $\mathrm{P} 1 \mathrm{M}$ ) showed the highest values of dissolved oxygen and surface velocity. Conductivity, width and depth of the medium increased toward the mouth. Several results are in accordance with the current literature dealing with community ecology of neotropical stream fish.
\end{abstract}

Keywords: Ichthyofauna, freshwater, diversity of species, spatial distribution.

TRINDADE, M.E.J., CETRA, M. \& JUCÁ-CHAGAS, R. Ictiofauna do RibeirãoLimoeiro, Bacia do Rio Cachoeira, BA. Biota Neotrop. 10(4): http://www.biotaneotropica.org.br/v10n4/pt/abstract?article+bn02010042010.

Resumo: Os riachos da Bacia do Leste caracterizam-se por serem pequenos, pouco complexos e com ictiofauna endêmica decorrente do isolamento geográfico. Neste contexto, foi realizado um estudo da distribuição das espécies e estrutura da comunidade de peixes do Ribeirão Limoeiro, BA ao longo de um gradiente espacial no período de um ano, utilizando-se a pesca elétrica como método de coleta. Foram coletadas 21 espécies totalizando 2264 indivíduos em quatro pontos: dois próximos da cabeceira (P1A e P1M), um no médio curso (P2) e outro próximo à sua foz (P3). Characiformes e Siluriformes foram as ordens mais representativas dentre as quatro registradas. Algumas espécies ocorreram exclusivamente na cabeceira (Astyanax sp. e Trichomycterus cf. bahianus) e outras próximo da foz (Leporinus sp. e Callichthys callichthys). Por outro lado, Characidium zebra, Parotocinclus cf. cristatus, Poecilia vivipara, Astyanax sp.1, Rhamdia sp. e Poecilia reticulata ocorreram em todos os pontos amostrados. Foi coletada uma espécie com "status" de vulnerável (Nematocharax venustus) e duas exóticas (Tilapia rendalli e Poecilia reticulata). Quanto à estrutura da comunidade, os valores dos índices de riqueza de "Margalef" e de diversidade de "Shannon" foram significativamente maiores no ponto P3, por outro lado, este ponto apresentou valores significativamente menores do índice de dominância de "Berger-Parker". O ponto com vegetação ripária preservada $(\mathrm{P} 1 \mathrm{M})$ apresentou menor valor médio da temperatura da água e os pontos de cabeceira (P1A e P1M) apresentaram os maiores valores médios de oxigênio dissolvido e velocidade superficial. Condutividade, largura e profundidade médias aumentaram da cabeceira para a foz. Vários resultados encontrados estão de acordo com a literatura atual que trata de ecologia de comunidades de peixes de riachos neotropicais.

Palavras-chave: Ictiofauna, água doce, diversidade de espécies, distribuição espacial. 


\section{Introdução}

A grande riqueza de espécies de peixes reflete-se também na sua diversidade morfológica e ecológica (Langeani, 2007). A maior parte dessa riqueza e diversidade encontra-se em águas tropicais (LoweMcConnell 1999), particularmente nas águas doces neotropicais, habitadas por 4.475 espécies válidas de peixes, podendo chegar a mais de 6.000 (dentre as 13.000 mundiais) se incluídas as novas espécies já reconhecidas por especialistas, porém ainda não descritas (Reis et al. 2003). Inventários recentes em ambientes de riachos e de cabeceiras no Alto Paraná, principalmente do Estado de São Paulo (e.g., Casatti et al. 2001, Castro \& Casatti 1997, Castro et al. 2003, 2004, 2005, Langeani et al. 2005), comprovam a ocorrência de uma fauna bastante diversificada, além de registrar a ocorrência de várias espécies exóticas e mostrar que cerca de 6 a 15\% das espécies referidas são novas (Castro et al. 2003, 2004, 2005).

A Bacia do Leste, onde se insere o riacho estudado, constitui um grupo de bacias hidrográficas, cujos rios correm exclusivamente em território brasileiro em direção ao Oceano Atlântico e estendemse dos Estados da Bahia ao Rio Grande do Sul (Menezes 1972). Caracterizam-se por apresentarem pequenas dimensões e baixa complexidade de seu sistema fluvial, sendo que os levantamentos ictiofaunísticos são poucos e não abrangem toda Bacia (Bizerril, 1994), sendo sua fauna caracterizada pelo elevado endemismo, decorrente do isolamento geográfico (Ribeiro 2006).

Segundo Nacif et al. (2003) a Bacia do Rio Cachoeira constitui um sistema socioecológico da maior importância para o Sul da Bahia, agrupa os principais municípios dessa região e apresenta marcante diversidade de áreas agrícolas que se distinguem por diferentes características naturais e sistemas de ocupação antrópica. No entanto, constata-se ainda uma carência de estudos detalhados deste sistema que possibilitem uma melhor definição de estratégias para um manejo sustentável dos diferentes ambientes desta bacia hidrográfica. Nesse contexto, foi realizado um estudo da composição e estrutura da comunidade de peixes do Ribeirão Limoeiro ao longo do gradiente longitudinal no período de um ano.

\section{Material e Métodos}

\section{1. Área de estudo}

A bacia hidrográfica do rio Cachoeira situa-se no sudeste da Bahia entre as coordenadas $14^{\circ} 42^{\prime}$ e $15^{\circ} 20^{\prime} \mathrm{S}$ e $39^{\circ} 01^{\prime}$ e $40^{\circ} 09^{\prime} \mathrm{W}$ e apresenta como limite as bacias dos Rios de Contas e Almada, ao norte, as bacias dos Rios Pardo e Una, ao sul; a bacia do Rio Pardo, a oeste e o Oceano Atlântico, a leste. O Rio Cachoeira tem cerca de $50 \mathrm{~km}$ de extensão e apresenta como principais afluentes os Rios Colônia, Salgado e Piabanhas. A área de drenagem da bacia corresponde a $4.600 \mathrm{~km}^{2}$ onde vivem aproximadamente 600.000 ha com atividades pecuaristas e de cultivo de cacau Theobroma cacao distribuídos em 12 municípios: Firmino Alves, Floresta Azul, Jussari, Itajú do Colônia, Ibicaraí, Ilhéus, Itabuna, Itapé, Itapetinga, Itororó, Lomanto Júnior e Santa Cruz da Vitória. O Rio Cachoeira nasce na serra de Ouricana numa altitude de 800 m na cidade de Itororó com o nome de Rio Colônia que encontra com o Rio Salgado e atinge seu patamar mais baixo na superfície litorânea do município de Ilhéus (Torres et al. 2001).

A bacia do Rio Cachoeira encontra-se inserida nas faixas climáticas do tipo Af, típico de florestas tropicais, com precipitação superior a $1000 \mathrm{~mm}$ anuais, bem distribuída durante todo o ano e temperatura média de $24{ }^{\circ} \mathrm{C}$, uma zona de clima de transição do tipo Am, caracterizada pela ocorrência de um período seco nos meses de agosto a setembro, compensado pelos totais pluviométricos elevados e temperaturas médias mensais elevadas e uma zona típica de clima tropical semi-úmido no sentido oeste próximo a região de planalto de Vitória da Conquista, com precipitação anual de $800 \mathrm{~mm}$ (Schiavetti et al. 2005).

O Ribeirão Limoeiro é um pequeno tributário permanente com cerca de $11 \mathrm{~km}$ de extensão, localizado próximo ao município de Floresta Azul. Os pontos de coleta foram definidos de acordo com a posição na bacia hidrográfica e acessibilidade ao local. Ponto 1 Aberto (P1A) (14 57' 57,6” S e 39 41' 42,9” W), Ponto 1 Mata (P1M) (14 ${ }^{\circ} 57^{\prime} 56,5^{\prime \prime} \mathrm{S}$ e $\left.39^{\circ} 41^{\prime} 49,7^{\prime \prime} \mathrm{W}\right)$, Ponto 2 (P2) (14 $56^{\prime} 45,5^{\prime \prime} \mathrm{S}$ e $\left.39^{\circ} 43^{\prime} 32,0^{\prime \prime} \mathrm{W}\right)$ e Ponto 3 (P3) ( $14^{\circ} 54^{\prime} 48,8^{\prime}$ ' S e $39^{\circ} 43^{\prime} 55,5^{\prime \prime} \mathrm{W}$ ) (Figura 1).

\section{Coleta e análise de dados}

Foram realizadas quatro amostragens de peixes em 2007 (março, junho, setembro e dezembro) utilizando a pesca elétrica, com metodologia modificada a partir de Suzuki et al. (1997) e Mazzoni et al. (2000). Esse método consiste em produzir um campo elétrico na água, passando uma corrente entre dois eletrodos submersos. $\mathrm{O}$ equipamento é constituído de um gerador com corrente continua (2000 W, $220 \mathrm{~V})$ e um transformador que permite a execução do trabalho em 220, 300, 400 e $500 \mathrm{~V}$, com uma corrente entre $1 \mathrm{~A}$ e 2A. A equipe de eletropesca foi constituída por um operador encarregado de ligar e desligar o aparelho e regular sua voltagem. Dois operadores vestidos com macacões de borracha isolante, equipados com eletrodos conectados a puçás que executavam movimentos de vai-e-vem, lateral, percorrendo um trecho de 75 a $100 \mathrm{~m}$ de extensão no sentido jusante-montante capturando com os puçás os peixes imobilizados pelo efeito da corrente elétrica. Uma quarta pessoa, também vestida com macacão de borracha, ficou responsável pelo manuseio dos fios, à medida que os coletores se deslocavam rio acima.

Em cada ponto amostrado foram obtidas as variáveis ambientais: largura (m), profundidade (m) e velocidade superficial da água (averiguada através do tempo de deslocamento de um objeto flutuante). A descrição dos pontos foi feita por meio da observação direta sobre o substrato, estrutura interna do canal e das margens ao longo do ponto amostrado.

Os peixes foram fixados em formol a $10 \%$ e preservados em etanol a $70 \%$ para posterior identificação. Exemplares testemunhos foram enviados para o Laboratório de Ictiologia do Museu de Ciências e Tecnologia da PUC/RS para confirmação taxonômica pelo Prof. Dr. Roberto Esser dos Reis e para o Laboratório de Ictiologia do Departamento de Zoologia da UFRGS para confirmação taxonômica pelo Prof. Dr. Luiz Roberto Malabarba.

Para cada amostragem foram calculados os índices de riqueza de Margalef, diversidade de Shannon, equabilidade de Pielou e dominância de Berger-Parker juntamente com o intervalo de confiança a 95\% calculado através do método de reamostragem "bootstrap". Foi utilizado o estimador de riqueza Chao 2 para estimar a riqueza esperada para a bacia do Ribeirão Limoeiro, juntamente com a variância. Todas estas medidas foram calculadas utilizando o software PAST (versão 1.68) (Hammer et al. 2001).

\section{Resultados}

De forma geral pode-se dizer que o P1A está inserido em um corpo d'água com traçado pouco sinuoso, sem mata ciliar e com pastagem no entorno, substrato arenoso composto por cascalhos, seixos e rochas expostas durante o seu curso. O P1M apresenta um traçado sinuoso com mata ciliar, substrato pedregoso composto por cascalhos e seixos e rochas expostas durante o seu curso, com troncos e galhos submersos. O P2, também possui um traçado sinuoso, com a mata ciliar comprometida em ambas as margens, substrato arenoso e apresenta uma combinação de rio corrente e remanso com águas mais 
lentas. O P3 possui um traçado pouco sinuoso, substrato pedregoso nos locais de corredeira e arenoso nos locais de menor velocidade, com rochas expostas no leito e apresenta maior combinação dos meso-habitats: rio corrente, corredeiras em menor extensão e poções nas margens e no meio do rio.

Foram coletados 2264 exemplares, pertencentes a 4 ordens, 11 famílias e 21 espécies de pequeno a médio porte, perfazendo um total de $7,6 \mathrm{~kg}$. Dentre as quatro ordens de peixes amostradas no Ribeirão Limoeiro, $82,72 \%$ das espécies capturadas pertence às ordens Characiformes e Siluriformes. A ordem Cyprinodontiformes teve sua maior frequência numérica no ponto $\mathrm{P} 1 \mathrm{~A}$ com $46,87 \%$. Os pontos $\mathrm{P} 2$ e P3 tiveram a maior frequência de ocorrência da ordem Perciformes, contribuindo com 43,21 e 50,75\%, respectivamente, além disso, vale ressaltar que no ponto $\mathrm{P} 1 \mathrm{~A}$ essa ordem não ocorreu.

As famílias mais representativas foram Characidae e Loricariidae que juntas contribuíram com o total de 58,39\% das espécies (Figura 2). A família Characidae teve sua maior frequência de ocorrência no ponto P1A com 40,64\%. Os pontos P2 e P3 tiveram a maior frequência de ocorrência da família Loricariidae, contribuindo com 50 e 30,36\% respectivamente (Figura 3).

As espécies Astyanax sp. e Trichomycterus cf. bahianus ocorreram apenas nos pontos $\mathrm{P} 1 \mathrm{~A}$ e P1M. As espécies Leporinus sp. e Callichthys callichthys ocorreram apenas no ponto P3. As espécies Characidium zebra, Parotocinclus cf. cristatus, Poecilia vivipara, Astyanax sp.1, Rhamdia sp. e Poecilia reticulata ocorreram em todos os pontos amostrados (Tabela 1).
A estimativa de riqueza de espécies para o Ribeirão Limoeiro foi de 21 espécies $\left(S_{\text {Chao }}=21,31 ; \mathrm{s}^{2}=0,67\right)$. Observou-se que o ponto P3 apresentou maior riqueza de espécies e os índices de riqueza, diversidade e equabilidade foram significativamente maiores que o P1A, que apresentou dominância significativamente maior que aquela encontrada no P3 (Tabela 1).

Os menores valores médios e desvio padrão da temperatura da água foram registrados no ponto que possui vegetação ripária (P1M). Os maiores valores médios de oxigênio dissolvido e velocidade foram registrados nos pontos mais elevados (P1A e P1M). Os valores de $\mathrm{pH}$ foram levemente alcalinos, independente do ponto de coleta e condutividade, largura e profundidade médias aumentaram da cabeceira para a foz (Tabela 2).

\section{Discussão}

O predomínio das ordens Characiformes e Siluriformes na ictiofauna do Ribeirão Limoeiro reflete o esperado para sistemas fluviais sul-americanos (Castro 1999, Lowe-McConnell 1999). Entretanto alguns trabalhos apresentam as ordens Siluriformes e Characiformes como as mais representativas em número de espécies seja na Mata Atlântica (Barreto \& Aranha 2005, Sabino \& Castro 1990) como no Alto Paraná (Castro \& Casatti 1997, Casatti 2005, Castro et al. 2004, Garutti 1988, Langeani et al. 2007, Pavanelli \& Caramaschi 1997).
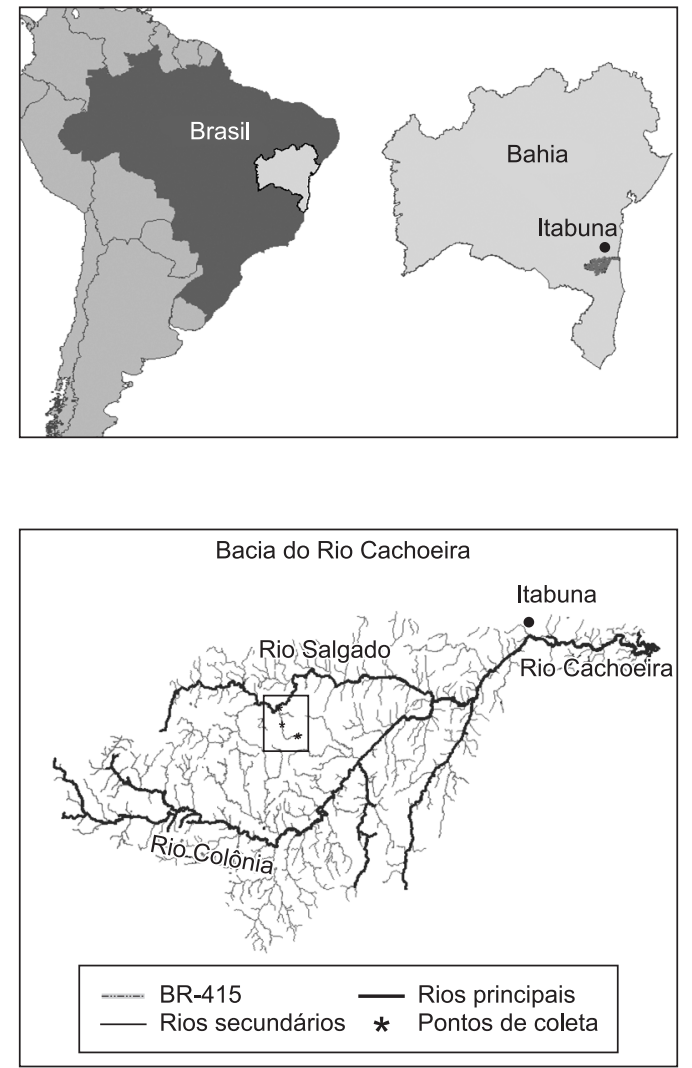

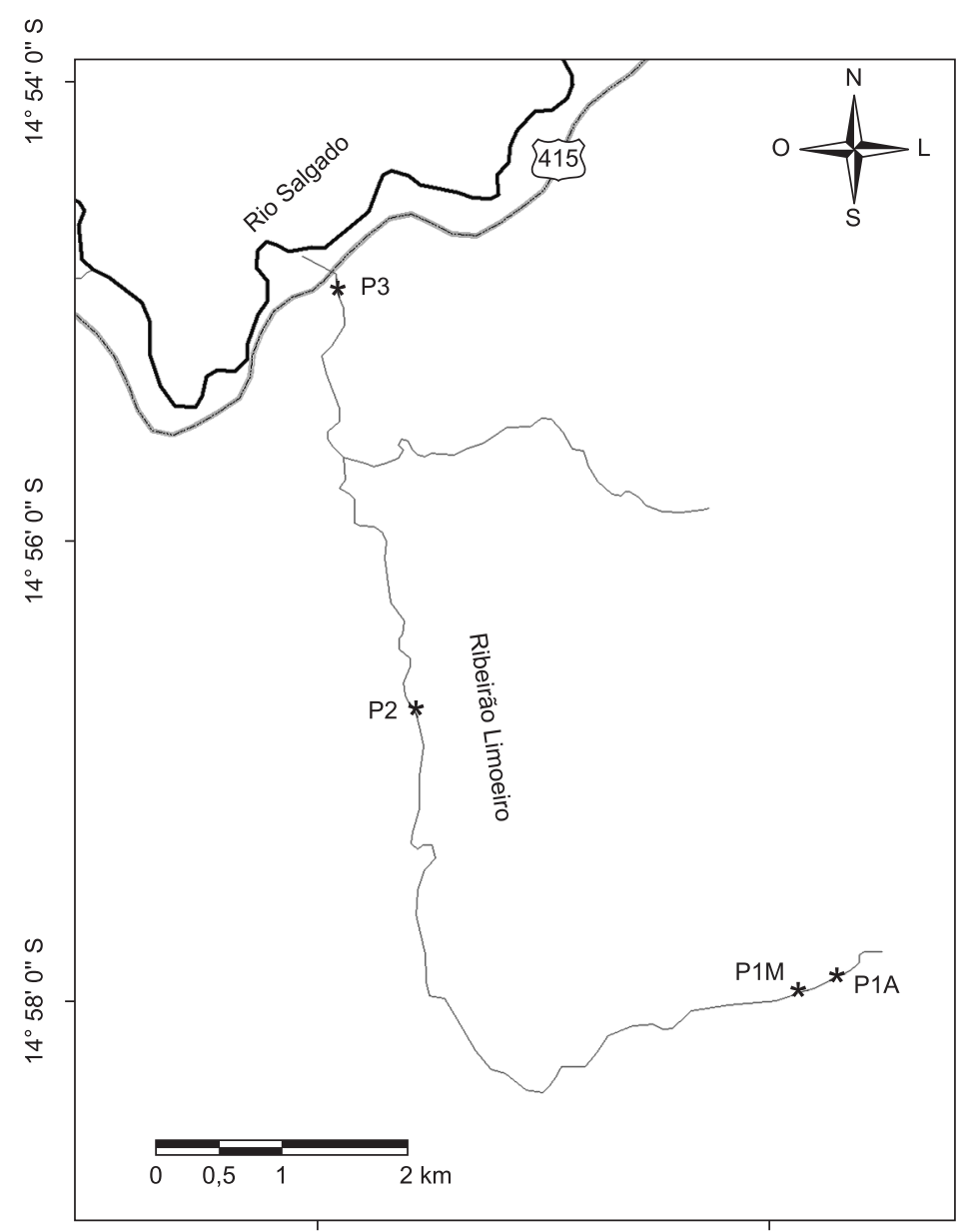

$39^{\circ} 44^{\prime} 0^{\prime \prime} \mathrm{W}$ $39^{\circ} 42^{\prime} 0^{\prime \prime} \mathrm{W}$

Figura 1. Bacia hidrográfica do Rio Cachoeira, BA, localização dos pontos de coleta no Ribeirão Limoeiro.

Figure 1. Cachoeira River basin, BA, location of points in the stream Limoeiro. 
Segundo Matthews (1998) uma interessante medida em estudo de comunidade de peixes, se refere ao número de famílias e ao número de espécies por família. Poucas famílias reúnem a maioria das espécies. No presente estudo verificou-se que Characidae e Loricariidae foram às famílias com maior número de espécies, fato também constatado por outros autores (Castro \& Casatti 1997, Miranda \& Mazzoni 2003, Uieda \& Barreto 1999).

Muitas das espécies registradas no Ribeirão Limoeiro, apresentam "status" taxonômico deficiente, como por exemplo, as espécies do gênero Astyanax, o que revela o pouco conhecimento da ictiofauna da região e reforça a necessidade de trabalhos taxonômicos. Recentemente foram descritas duas espécies novas de Astyanax, no estado da Bahia: Astyanax vermilion, no Rio Almada e Rio Cachoeira, e Astyanax burgerai, no Rio Almada (Zanata \& Camelier 2009). Entretanto poucos são os trabalhos de cunho ecológico/naturalístico envolvendo ambientes fluviais de pequeno e médio porte (Castro, 1999). Esse fato explica os problemas frequentes relativos aos limites taxonômicos de espécies de riachos da região Neotropical (Pavanelli \& Caramaschi 1997).

Gêneros tais como Astyanax e Hypostomus estão entre os táxons neotropicais mais especiosos, o que resulta em diversos problemas quanto aos reais limites taxonômicos de suas espécies (Miranda \& Mazzoni 2003). Lowe-McConnell (1999) afirma que o estudo de ecologia de peixes em uma área tão vasta e pouco explorada como a

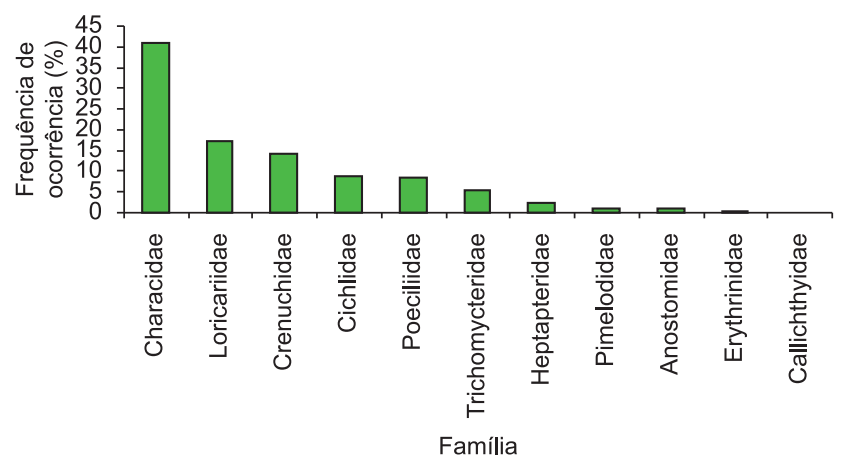

Figura 2. Frequência numérica (\%) das famílias de peixes amostradas no Ribeirão Limoeiro.

Figure 2. Numerical frequency (\%) of families of fish sampled in the stream Limoeiro.

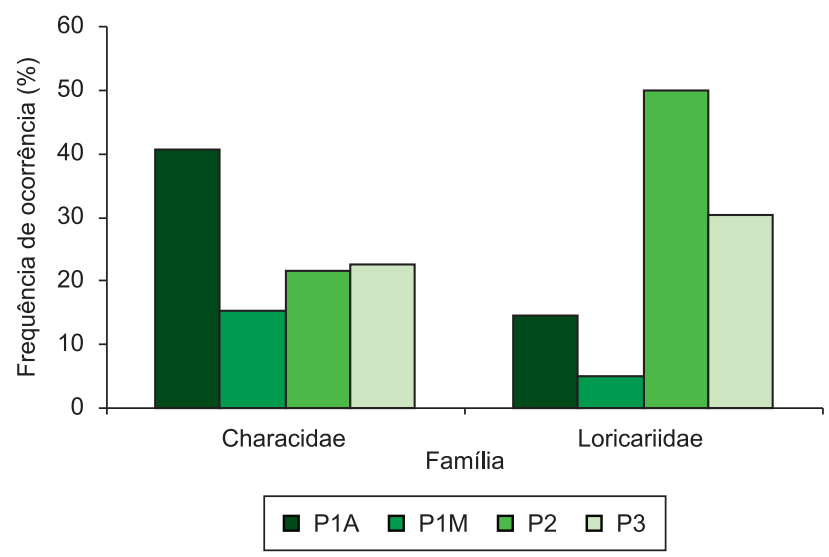

Figura 3. Frequência de ocorrência (\%) das famílias de peixes representadas em cada ponto amostrado do Ribeirão Limoeiro.

Figure 3. Frequency of occurrence (\%) of families of fish represented in each point sampled the stream Limoeiro. região Neotropical, é uma tarefa dificultada pela presença de muitas espécies, várias das quais muito semelhante entre si, tornando difícil sua identificação, fato que explica os morfotipos encontrados no presente trabalho.

Menezes et al. (2007) afirmam que mais de cem espécies são atribuídas ao gênero Hypostomus, mas o conhecimento taxonômico deste gênero é muito pobre. Dados da biologia de algumas espécies como, Hypostomus obtusirostris do Rio Cubatão (Santa Catarina), Hypostomus punctatus (Rio de Janeiro), Hypostomus brevicauda (Bahia) e Hypostomus cf. unae do Rio Una (Bahia) presente no Ribeirão Limoeiro, são conhecidas apenas por meio de suas respectivas descrições originais.

Com relação aos tricomicterídeos, estes representam um dos grupos menos conhecidos. Segundo Menezes et al. (2007) a espécie Trichomycterus cf. bahianus, por exemplo, tem sua distribuição conhecida apenas na bacia do Rio Una (BA) sendo sua ecologia e estado de conservação desconhecidos. No presente estudo essa espécie esteve presente apenas nos pontos de cabeceira. Oliveira \& Bennemann (2005) sugerem que a presença de espécies do gênero Trichomycterus indica que o ambiente encontra-se pouco degradado.

Vale ressaltar a presença da espécie Nematocharax venustus. Segundo Menezes et al. (2007) o estado de conservação dessa espécie encontra-se vulnerável, sua distribuição é conhecida da bacia do Rio do Braço ao sul no Rio Una e partes centrais e leste do Rio Jequitinhonha, Sul da Bahia e partes adjacentes de Minas Gerais.

Outro aspecto a ser considerado é a presença de espécies exóticas como Tilapia rendalli e Poecilia reticulata. Segundo Vieira \& Shibatta (2007) P. reticulata, é uma espécie de interesse aquarístico que possui capacidade de resistência a ambientes que sofreram alterações antrópicas, pois se alimenta de detritos variados, sendo bastante oportunista. Oliveira \& Bennemann (2005) verificaram que no Ribeirão Cambé (PR) o maior número de espécies se deve, em parte, a presença de espécies introduzidas. Dentre os efeitos das alterações longitudinais na estrutura das comunidades, o aumento da diversidade da comunidade lótica em direção a foz, em suas diferentes formas (riqueza, equabilidade, entre outras) é um dos padrões mais evidentes (Súarez \& Petrere Jr. 2006). Vários autores têm associado o aumento da diversidade ao longo do rio com o aumento da heterogeneidade de hábitats (Langeani et al. 2005), o qual, por sua vez, é relacionado principalmente a um aumento na vazão e na disponibilidade de abrigos (Garutti 1988, Uieda \& Barreto 1999, Braga \& Andrade 2005, Cetra \& Petrere Jr. 2006). Como sugerido por Bojsen \& Barriga (2002), a relação positiva entre a riqueza de espécies e tamanho do hábitat não é somente associada com o esforço amostral. No Ribeirão Limoeiro pôde-se verificar que a riqueza, diversidade e equabilidade aumentaram em direção à jusante, sendo significativamente maior no ponto $\mathrm{P} 3$ em relação aos demais pontos amostrados, coincidindo com resultados encontrados por Abes \& Agostinho (2001) onde a riqueza, diversidade e equabilidade aumentaram em direção aos segmentos inferiores. Magurran \& Phillip (2001) constataram em dois riachos em Trinidad que a assembléia de peixe estudada mostrou notável relação entre o índice de dominância e riqueza de espécies, sendo que, no local onde a riqueza de espécies aumentou, o índice de dominância tornou-se menor. Tal inferência pode ser confirmada pela observação dos índices de equabilidade e dominância, que no ponto P3 foram, respectivamente, maior e menor em relação ao ponto P1A. Ressaltase que o ponto P3 apresentou os maiores valores de profundidade, largura e condutividade e os menores valores de temperatura, oxigênio dissolvido, $\mathrm{pH}$ e velocidade superficial.

Um parâmetro importante que deve ser levado em consideração na estruturação da ictiofauna em relação à distribuição longitudinal é a cobertura vegetal (Bojsen \& Barriga 2002). Segundo 
Tabela 1. Composição específica, abundância total $(\mathrm{N})$, riqueza de espécies $(\mathrm{S})$, abundância $(\mathrm{N})$, índice de riqueza de Margalef $\left(\mathrm{D}_{\mathrm{Mg}}\right)$, índice de diversidade de Shannon (H'), índice de equabilidade (J') e índice de dominância de Berger-Parker (d), (intervalo de confiança de 95\%) para cada ponto amostrado do Ribeirão Limoeiro, durante todo período estudado

Table 1. Species composition, total abundance $(\mathrm{N})$, species richness $(\mathrm{S})$, abundance $(\mathrm{N})$, Margalef's index of richness $\left(\mathrm{D}_{\mathrm{Mg}}\right)$, Shannon's index of diversity $\left(\mathrm{H}^{\prime}\right)$, equitability index (J') and dominance index of Berger-Parker (d), (confidence interval 95\%) in each section of stream Limoeiro throughout the study period.

\begin{tabular}{|c|c|c|c|c|c|}
\hline \multirow[t]{2}{*}{ Espécies } & \multicolumn{4}{|c|}{ Pontos de coleta } & \multirow{2}{*}{$\begin{array}{c}\text { Total } \\
\mathbf{N}\end{array}$} \\
\hline & P1A & P1M & $\mathbf{P 2}$ & $\mathbf{P 3}$ & \\
\hline Astyanax sp. & 1 & 1 & 0 & 0 & 376 \\
\hline Characidium zebra Eigenmann, 1909 & 1 & 1 & 1 & 1 & 322 \\
\hline Paratocinclus cf. cristatus Garavello, 1977 & 1 & 1 & 1 & 1 & 213 \\
\hline Geophagus brasiliensis (Quoy \& Gaimard, 1824) & 0 & 1 & 1 & 1 & 195 \\
\hline Nematocharax venustus Weitzman, Menezes \& Britski, 1986 & 0 & 0 & 1 & 1 & 193 \\
\hline Poecilia vivipara Bloch \& Schneider, 1801 & 1 & 1 & 1 & 1 & 160 \\
\hline Hypostomus cf. unae (Steindachner, 1878) & 1 & 0 & 1 & 1 & 166 \\
\hline Astyanax sp.1 & 1 & 1 & 1 & 1 & 145 \\
\hline Trichomycterus cf. bahianus Costa, 1992 & 1 & 1 & 0 & 0 & 126 \\
\hline Astyanax sp.3 & 0 & 0 & 1 & 1 & 90 \\
\hline Hyphessobrycon sp. & 1 & 0 & 1 & 1 & 90 \\
\hline Rhamdia sp. & 1 & 1 & 1 & 1 & 54 \\
\hline Astyanax bimaculatus (Linnaeus, 1758) & 0 & 0 & 1 & 1 & 34 \\
\hline Poecilia reticulata Peters, 1859 & 1 & 1 & 1 & 1 & 32 \\
\hline Pimelodella sp. & 0 & 0 & 1 & 1 & 21 \\
\hline Leporinus sp. & 0 & 0 & 0 & 1 & 20 \\
\hline Hypostomus sp. & 0 & 0 & 1 & 1 & 13 \\
\hline Hoplias malabaricus (Bloch, 1794) & 0 & 0 & 1 & 1 & 7 \\
\hline Tilapia rendalli (Boulenger, 1897) & 0 & 0 & 0 & 1 & 4 \\
\hline Astyanax sp.2 & 0 & 0 & 1 & 0 & 2 \\
\hline Callichthys callichthys (Linnaeus, 1758) & 0 & 0 & 0 & 1 & 1 \\
\hline$S$ & 10 & 9 & 16 & 18 & 21 \\
\hline $\mathrm{N}$ & 636 & 297 & 699 & 632 & 2264 \\
\hline $\mathrm{D}_{\mathrm{Mg}}$ & $\begin{array}{l}1,39 \\
(1,39-1,39)\end{array}$ & $\begin{array}{l}1,40 \\
(1,40-1,40)\end{array}$ & $\begin{array}{l}2,29 \\
(1,83-2,29)\end{array}$ & $\begin{array}{l}2,64 \\
(2,33-2,64)\end{array}$ & $\begin{array}{l}2,59 \\
(2,33-2,59)\end{array}$ \\
\hline $\mathrm{H}^{\prime}$ & $\begin{array}{l}1,83 \\
(1,75-1,90)\end{array}$ & $\begin{array}{l}1,86 \\
(1,75-1,93)\end{array}$ & $\begin{array}{l}1,82 \\
(1,74-1,88)\end{array}$ & $\begin{array}{l}2,41 \\
(2,34-2,46)\end{array}$ & $\begin{array}{l}2,56 \\
(2,52-2,58)\end{array}$ \\
\hline J' & $\begin{array}{l}0,79 \\
(0,76-0,82)\end{array}$ & $\begin{array}{l}0,84 \\
(0,79-0,88)\end{array}$ & $\begin{array}{l}0,65 \\
(0,64-0,70)\end{array}$ & $\begin{array}{l}0,84 \\
(0,82-0,87)\end{array}$ & $\begin{array}{l}0,84 \\
(0,83-0,87)\end{array}$ \\
\hline d & $\begin{array}{l}0,41 \\
(0,38-0,46)\end{array}$ & $\begin{array}{l}0,36 \\
(0,31-0,42)\end{array}$ & $\begin{array}{l}0,28 \\
(0,25-0,31)\end{array}$ & $\begin{array}{l}0,16 \\
(0,15-0,19)\end{array}$ & $\begin{array}{l}0,16 \\
(0,15-0,18)\end{array}$ \\
\hline
\end{tabular}

Tabela 2. Valores médios e desvio padrão para as variáveis físicas e químicas da água nos diferentes pontos do Ribeirão Limoeiro, BA, Brasil.

Table 2. Mean values and standard deviation for the physical and chemical parameters of water in different points of the stream Limoeiro, BA, Brazil.

\begin{tabular}{lrrrr}
\hline Variáveis físicas e químicas & \multicolumn{1}{c}{ P1A } & P1M & P2 & P3 \\
\hline Temperatura da água $\left({ }^{\circ} \mathrm{C}\right)$ & $24,52 \pm 2,12$ & $22,99 \pm 1,44$ & $24,45 \pm 2,36$ & $25,40 \pm 1,73$ \\
Oxigênio dissolvido $\left(\mathrm{mg} . \mathrm{L}^{-1}\right)$ & $7,24 \pm 1,20$ & $7,12 \pm 1,12$ & $6,34 \pm 1,94$ & $6,43 \pm 2,08$ \\
pH & $7,19 \pm 0,36$ & $7,18 \pm 0,23$ & $7,30 \pm 0,21$ & $7,77 \pm 0,15$ \\
Condutividade $\left(\mu \mathrm{S} . \mathrm{cm}^{-1}\right)$ & $104,25 \pm 5,79$ & $103,17 \pm 4,99$ & $314,50 \pm 39,14$ & $789,67 \pm 126,78$ \\
Largura $(\mathrm{m})$ & $1,54 \pm 0,73$ & $1,99 \pm 0,50$ & $2,84 \pm 0,88$ & $5,22 \pm 0,40$ \\
Profundidade $(\mathrm{m})$ & $0,21 \pm 0,07$ & $0,35 \pm 0,12$ & $0,46 \pm 0,07$ & $0,61 \pm 0,12$ \\
Velocidade $(\mathrm{m} / \mathrm{s})$ & $0,50 \pm 0,12$ & $0,47 \pm 0,08$ & $0,33 \pm 0,15$ & $0,40 \pm 0,08$ \\
\hline
\end{tabular}


Menezes et al. (2007) a diversidade das espécies é reduzida nos riachos de áreas desmatadas em consequência de atividades agrícolas, o que favorece maior intensidade luminosa, crescimento de plantas aquáticas vasculares, especialmente plantas emersas, devido à maior disponibilidade de nutrientes (fertilizantes) e, em alguns casos, a existência de grande quantidade de sedimento devido à erosão.

Cetra \& Petrere Jr. (2007) verificaram a associação entre as assembléias de peixes e a mata ciliar na bacia do Rio Corumbataí (SP) e constataram maior riqueza de espécies nos locais com maior cobertura vegetal e mata ciliar preservada. Resultados semelhantes foram encontrados por Sarmento-Soares et al. (2007) para a fauna de peixes na bacia do Rio Peruípe, extremo Sul da Bahia. Por outro lado, Bojsen \& Barriga (2002), encontraram um maior riqueza de espécies nos locais desmatados em relação aos locais de cobertura vegetal, porém, não encontraram diferença significativa para a riqueza média das espécies entre os pontos. Os autores sugerem que essa maior riqueza nos locais desmatados pode estar relacionada ao aumento na densidade de macroinvertebrados. Segundo Angermeier \& Karr (1983) as fontes de alimento autóctone têm mantido grande proporção de espécies de peixes em ambientes desmatados. Porém, os indicadores da estrutura da comunidade de peixes do Ribeirão Limoeiro não apontaram diferença significativa entre os dois pontos P1A e P1M, a não ser quanto à abundância que foi muito maior no P1A.

\section{Agradecimentos}

Somos gratos a Karen, Tito, Gabriel, Márcio, Polli, Camilla, Leonardo, Fábio e Alberto pelo auxilio em campo. Ao Prof. Dr. Luiz Roberto Malabarba (Universidade Federal do Rio Grande do Sul) e ao Prof. Dr. Roberto Esser dos Reis (Pontifícia Universidade Católica do Rio Grande do Sul) pela confirmação taxonômica dos exemplares estudados. Ao Programa de Pós-graduação em Sistemas Aquáticos Tropicais pelo apoio durante a realização deste trabalho através do projeto SATCAP - Capacidade suporte da bacia do Rio Cachoeira e área costeira adjacente frente à sustentabilidade ambiental dos recursos hídricos (CNPq CTHIDRO 14/2005 Processo 133343/20065). A Fundação de Amparo a Pesquisa do Estado da Bahia (FAPESB) pelo apoio financeiro.

\section{Referências Bibliográficas}

ABES, S.S. \& AGOSTINHO, A.A. 2001. Spatial patterns in fish distributions and structure of the ichthyocenosis in the Água Nanci stream, upper Paraná River basin, Brazil. Hydrobiologia 445:217-227.

ANGERMEIER, P.L. \& KARR, J.R. 1983. Fish communities along environmental gradients in a system of tropical streams. Env. Bio. Fish. 9(2):117-135

BARRETO, A. P. \& ARANHA, J.M. 2005. Assembléia de peixes de um riacho da Floresta Atlântica: composição e distribuição espacial (Guaraqueçaba, Paraná, Brasil). Acta Sci. Biol. Sci. 27(2):153-160.

BIZERRIL, C.R.S.F. 1994. Análise taxonômica e biogeográfica da ictiofauna de água doce do leste brasileiro. Acta Leopoldensia 16:51-80.

BOJSEN, B.H. \& BARRIGA, R. 2002. Effects of deforestation on fish community structure in Ecuadorian Amazon streams. Freshw. Biol. 47:2246-2260.

BRAGA, F.M.S. \& ANDRADE, P.M. 2005. Distribuição de peixes na microbacia do Ribeirão Grande, serra da Mantiqueira Oriental, São Paulo, Brasil. Iheringia, Ser. Zool. 95(2):121-126.

CASATTI, L. 2005. Fish assemblage structure in a first order stream, Southeastern Brazil: longitudinal distribution, seasonality, and microhabitat diversity. Biota Neotrop. 5(1) http://www.biotaneotropica. org.br/v5n1/pt/abstract?article+BN02505012005 (ultimo acesso em 10/09/2007).
CASATTI, L., LANGEANI, F. \& CASTRO, R.M.C. 2001. Peixes de riacho do parque Estadual Morro do Diabo, bacia do alto Rio Paraná, SP. Biota Neotrop. 1(1) http://www.biotaneotropica.org.br/v1n12/pt/ abstract?+BN00201122001 (ultimo acesso em 10/09/2007).

CASTRO, R.M.C. \& CASATTI, L. 1997.The fish fauna from a small forest stream of the upper Paraná river basin, Southeastern Brazil. Ichthyol. Explor. Freshw. 7(4):337-352.

CASTRO, R.M.C. 1999. Evolução da ictiofauna de riachos sul-americanos: padrões gerais e possíveis processos causais. In Ecologia de peixes de riachos (E.P. Caramaschi, R. Mazozoni, P.R. Peres-neto, ed). Computer \& Publish Editoração, Rio de Janeiro, p.139-155.

CASTRO, R.M.C., CASATTI, L., SANTOS, H.F., FERREIRA, K.M., RIBEIRO, A.C., BENINE, R.C., DARDIS, G.Z.P., MELO, A.L.A., STOPIGLIA, R., ABREU, T.X., BOCKMANN, F.A., CARVALHO, M., GIBRAN, F.Z. \& LIMA, F.C.T. 2003. Estrutura e composição da ictiofauna de riachos do Rio Paranapanema, Sudeste do Brasil. Biota Neotrop. 3(1) http://www.biotaneotropica.org.br/v3n1/pt/ abstract?article+BN01703012003 (úrltimo acesso em 15/09/2007).

CASTRO, R.M.C., CASATTI, L., SANTOS, H.F., MELO, A.L.A., MARTINS, L.S.F., FERREIRA, K.M., GIBRAN, F.Z., BENINE, R.C., CARVALHO, M., RIBEIRO, A.C., ABREU, T.X., BOCKMANN, F.A., PELIÇÃO, G.Z., STOPIGLIA, R. \& LANGEANI, F. 2004. Estrutura e Composição da ictiofauna de riachos da bacia do rio Grande no Estado de São Paulo, Sudeste do Brasil. Biota Neotrop. 4(1) http://www.biotaneotropica. org.br/v4n1/pt/abstract?article+BN01704012004 (último acesso em 18/09/2007).

CASTRO, R.M.C., CASATTI, L., SANTOS, H.F., VARI, R.P., MELO, A.L.A., MARTINS, L.S.F., ABREU, T.X., BENINE, R.C., GIBRAN, F.Z., RIBEIRO, A.C., BOCKMANN, F.A., CARVALHO, M., PELIÇÃO, G.Z., FERREIRA, K.M., STOPIGLIA, R. \& AKAMA, A. 2005. Structure and composition of the stream ichthyofauna of four tributary rivers of the upper Rio Paraná basin, Brazil. Ichthyol. Explor. Freshwaters 16(3):193-214.

CETRA, M. \& PETRERE Jr., M. 2006. Fish assemblage structure of the Corumbatai river basin, São Paulo State, Brazil: characterization and anthropogenic disturbances. Brazil. J. Biol., 66(2A):431-439.

CETRA, M. \& PETRERE Jr., M. 2007. Associations between fish assemblages and riparian vegetation in the Corumbataí River Basin (SP). Brazil. J. Biol. 67(2):191-195.

GARUTTI, W. 1988. Distribuição Longitudinal da ictiofauna em um córrego da região noroeste do Estado de São Paulo, bacia do Rio Paraná. Rev. Brasil. Biol. 48(4):747-759.

HAMMER O., HARPER, D.A.T. \& RYAN, P.D. 2001. Past: Palaeontological statistics software package for education and data analysis. Palaeontologia Eletronica 4(1):9.

LANGEANI, F., CASATTI, L., GAMEIRO, H.S., CARMO, A.B. \& ROSSAFERES, D.C. 2005. Riffle and pool fish communities in a large stream of southeastern Brazil. Neotrop. Ichthyol. 3(2):305-311.

LANGEANI, F., CASTRO, R.M.C., OYAKAWA, O.T., SHIBATTA, O.A., PAVANELLI, C.S. \& CASATTI, L. 2007. Diversidade da ictiofauna do alto Rio Paraná: composição atual e perspectivas futuras. Biota Neotrop. 7(3): http://www.biotaneotropica.org.br/v7n3/pt/ abstract?article+bn03407032007 (último acesso em 12/04/2008).

LOWE-McCONNELL, R.H. 1999. Estudos ecológicos de comunidades de peixes tropicais. Edusp, São Paulo, 536p.

MAGURRAN, A.E. \& PHILLIP, D.A.T. 2001. Implications of species loss in freshwater fish assemblages. Ecography. 24:645-650.

MATTHEWS, W.J. 1998. Patterns in freshwater fish ecology. Chapman \& Hall, New York, 756p.

MAZZONI, R., FENERICH-VERANI, N., CARAMASCHI, E.P. 2000. Electrofishing as a sampling technique for coastal stream fish populations and communities in the Southeast of Brazil. Rev. Brasil. Biol. 60(2):205-216. 
MENEZES, N.A. 1972. Distribuição e origem da fauna de peixes de água-doce das grandes bacias fluviais do Brasil. In Poluição e piscicultura (Comissão Internacional da Bacia Paraná-Uruguai). Faculdade de Saúde Pública da USP, Instituto de Pesca, São Paulo, p.79-108.

MENEZES, N.A., WEITZMAN, S.H., OYAKAWA, O.T., LIMA, F.C.T., CASTRO, R.M.C. \& WEITZMAN, M.J. 2007. Peixes de água doce da Mata Atlântica: lista preliminar das espécies e comentários sobre conservação de peixes de água doce neotropicais. Museu de Zoologia, Universidade de São Paulo, São Paulo, 408p.

MIRANDA, J.C. \& MAZZONI, R. 2003. Composição da ictiofauna de três riachos do alto rio Tocantins, GO. Biota Neotrop. 3(1) http://www. biotaneotropica.org.br/v3n1/pt/abstract?article+BN00603012003 (último acesso em 18/10/ 2007).

NACIF, P.G.S., COSTA, L.M., SAADI, A., FERNANDES FILHO, E.I., KER, J.C., COSTA, O.V. \& MOREAU, M.S. 2003. Ambientes naturais da bacia hidrográfica do rio Cachoeira. Cruz das Almas, Brasil.

OLIVEIRA, D.C. \& BENNEMANN, S.T. 2005. Ictiofauna, recursos alimentares e relações com as interferências antrópicas em um riacho urbano no Sul do Brasil. Biota Neotrop. 5(1): http: //www.biotaneotropica. org.br/v5n1/pt/abstract?article+BN02905012005 (último acesso em 08/09/ 2007).

PAVANELLI, C.S. \& CARAMASCHI, E.P. 1997. Composition of the ichthyofauna of two small tributaries of the Paraná River, Porto Rico, Paraná State, Brazil. Ichthyol. Explor. Freshw. 8:32-31

REIS, R.E., KULLANDER, S.O. \& FERRARIS Jr., C.J. (org.). 2003. Check list of the freshwater fishes of South and Central America. EDIPUCRS, Porto Alegre.

RIBEIRO, A.C. 2006.Tectonic history and the biogeography of the freshwater fishes from the coastal drainages of eastern Brazil: an example of fauna evolution associated with a divergent continental margin. Neotrop. Ichthyol. 4(2):225-246.
SABINO, J. \& CASTRO, R.M.C. 1990. Alimentação, período de atividade e distribuição espacial dos peixes de um riacho de Floresta Atlântica (Sudeste do Brasil). Rev. Brasil. Biol. 50(1):23-36.

SARMENTO-SOARES, L.M., MAZZONI, R. \& MARTINS-PINHEIRO, R.F. 2007. A fauna de peixes na bacia do Rio Peruípe, extremo Sul da Bahia. Biota Neotrop. 7(3) http://www.biotaneotropica.org.br/v7n3/pt/abstract ?inventory+bn02107032007 (último acesso em 05/03/ 2008).

SCHIAVETTI, A., SCHILLING, A.C. \& OLIVEIRA, H.T. 2005. Caracterização sócio-ambiental da bacia hidrográfica do Rio Cachoeira Sul da Bahia, Brasil. In Conceitos de bacias hidrográficas teorias e aplicações (A. Schiavetti \& A.F.M. Camargo). Editus, Ilhéus, BA, 293p.

SÚAREZ, Y.R. \& PETRERE Jr., M. 2006. Gradientes de diversidade nas comunidades de peixes da bacia do Rio Iguatemi, Mato Grosso do Sul, Brasil. Iheringia, Sér. Zool. 96(2):197-204.

SUZUKI, H.I., PAVANELLI, C.S., FUGI, R., BINI, L.M. \& AGOSTINHO, A. A. 1997. Ictiofauna de quatro tributários do reservatório de Segredo. In Reservatório de Segredo - bases ecológicas para o manejo (A.A. Agostinho \& L.C. Gomes, ed). EDUEM, Maringá, p.259-273.

TORRES, M.L.M., REGO, N.C., GEUZ, F., LEVY, M.C. \& MOREAU, M. 2001. Programa de recuperação das bacias dos rios Cachoeira e Almada -Diagnóstico Regional. Núcleo de bacias hidrográficas da UESC, Superintendência de Recursos Hídricos do Estado da Bahia.

UIEDA, V.S. \& BARRETO, M.G. 1999. Composição da ictiofauna de quatro trechos de diferentes ordens do Rio Capivara, bacia do Tietê, Botucatu, São Paulo. Rev. Brasil. Zool. 1(1):55-67.

VIEIRA, D.B. \& SHIBATTA, O.A. 2007. Peixes como indicadores da qualidade ambiental do Ribeirão Esperança município de Londrina, Paraná, Brasil. Biota Neotropica. 7(1) http://www.biotaneotropica.org.br/v7n1/pt/ abstract?article+bn01407012007 (último acesso em 05/10/ 2007).

ZANATA, A.M. \& CAMELIER, P. 2009. Astyanax vermilion and Astyanax burgerai: new characid fishes (Ostariophysi: Characiformes) from Northeastern Bahia, Brazil. Neotrop. Ichthyol. 7(2):175-184.

Recebido em 29/12/2009

Versão reformulada recebida em 17/09/2010

Publicado em 21/10/2010 
